\title{
Wheat Bran Cereal, Human Gut Bacteria and Subjective Wellbeing
}

\author{
Andrew P Smith ${ }^{1}$, Eddie Deaville ${ }^{2} \&$ Glenn R Gibson $^{2}$ \\ ${ }^{1}$ School of Psychology, Cardiff University, 63 Park Place, Cardiff CF10 3AS, UK \\ ${ }^{2}$ Food Microbial Sciences Unit, Department of Food and Nutritional Sciences, The University of Reading, \\ Whiteknights, Reading RG6 6AP, UK \\ Correspondence: Andrew P Smith, School of Psychology, Cardiff University, 63 Park Place, Cardiff CF10 3AS, \\ UK. E-mail: smithap@ cardiff.ac.uk
}

Received: November 4, 2017

Accepted: November 24, 2017 Online Published: March 17, 2018

doi:10.5539/jfr.v7n3p8

URL: https://doi.org/10.5539/jfr.v7n3p8

\begin{abstract}
Research has shown that consumption of high fiber breakfast cereal is associated with improved subjective well-being, especially increased energy. One possible explanation of these results is through metabolism by gut bacteria and concomitant production of metabolites that influence psychological and gastrointestinal (GI) welfare. This was examined in the present study to determine whether consumption of wheat bran could modulate the composition of the GI microbiota. This human volunteer study (20 volunteers) involved the comparison of three breakfast cereals, All-Bran, Bran Flakes and Cornflakes $(60 \mathrm{~g} / \mathrm{d})$. The study involved a 14-day baseline phase (no breakfast cereals) and an eight-week experimental phase. Each cereal was consumed for 14 days. A seven-day washout period (no cereals) was carried out between each successive cereal condition. Faecal samples were collected every seven days. Enumeration of predominant faecal bacterial populations (bacteroides, bifidobacteria, clostridia, lactobacilli and eubacteria) was carried out using the culture independent fluorescent in situ hybridisation (FISH) technique. Faecal short chain fatty acid content was also determined. The volunteers completed a battery of questionnaires to assess fatigue/energy, subjective mood, physical and mental health, bowel function and fiber intake. The results showed that in general there was no overall significant effect of breakfast cereal type on the faecal bacterial populations studied. There was also no major effect of breakfast type on short chain fatty acid content. The high-fiber conditions (All-Bran and Bran Flakes) were associated with less fatigue, a significant reduction in cognitive difficulties, looser stools, more motions and feeling more energised.
\end{abstract}

Keywords: prebiotic, fiber, wheat bran, fatigue, cognitive difficulties, clostridia, SCFA.

\section{Introduction}

There is considerable evidence that constipation and other disorders of bowel functioning are significant causes of morbidity (Garrow \& James, 1993). Changes in dietary pattern are one of the main causes of the high prevalence of these disorders, although the aetiology can be complex (Taylor, 1990). Dietary fiber can improve bowel functioning and wheat bran is often the fiber of choice for the treatment of constipation because of its ability to increase faecal bulk (Gray, 1995). Indeed, the addition of wheat bran to the diet prevents constipation in up to $60 \%$ of elderly patients (Hull, Greco \& Brooks, 1980). Overall, therefore, there is ample evidence to show that wheat bran has beneficial effects on bowel function.

Bowel functioning is associated with other aspects of health. For example, when a person is stressed their bowel habit often changes. Patients with constipation have increased psychopathology (Belsey, Greenfield, Candy, \& Geraint, 2010). These findings have been confirmed in an epidemiological study of a community sample of 15 000 volunteers (Smith, 2011). Smith, Bazzoni, Beale, Elliott-Smith and Tilley (2001) tested the hypothesis that fiber regulates the digestive system which then leads to a state of improved psychological/physical wellbeing. Initial cross-sectional analyses examined associations between these variables. An intervention study was then carried out to determine whether increasing fiber intake, by consuming All-Bran or Bran Flakes, was associated with changes in bowel functioning and physical/mental health. The cross-sectional analyses (Smith et al., 2001, Smith, 2005) showed that high fiber intake was associated with better physical and mental health. Similarly, digestive problems were associated with impaired health and well-being, with the profile of effects depending on the digestive symptoms reported. The intervention study showed that both consumption of All-Bran and Bran 
Flakes led to a change in bowel functioning (more digestive problems in the first week, fewer in the second) and increased energy (reduced fatigue). These effects on fatigue were consistent over time. Results suggested that increased dietary fiber from wheat bran cereals reduced fatigue. This effect did not depend on the removal of symptoms of bowel dysfunction. There is now interest in the use of foods that are able to modulate the composition of the gastrointestinal (GI) microflora, such as lactobacilli and bifidobacteria, of humans in a manner that improves health. Lactobacilli and bifidobacteria are believed to improve resistance to GI infections by inhibiting the growth of harmful bacteria, reducing cholesterol levels, improving the immune response and producing vitamins (Gibson, 1998). Many dairy products now have added live additions of health promoting bacteria as probiotics (Hill et al., 2014), whilst the fermentation of certain carbohydrates (prebiotics) can selectively enhance beneficial components of the gut microbiota, such as lactobacilli and bifidobacteria (Gibson \& Roberfroid, 1995). While the former approach has been widely advocated, it is likely that a greater number of food products may have prebiotic effects. Moreover, virtually any carbohydrate-based material/product can be fortified with prebiotics (e.g. inulin), cereals being a particularly attractive food vehicle. There is also great interest in determining the 'intrinsic' prebiotic potential of products such as cereals.

The objective of the present study, therefore, was to determine the effect of All-Bran and Bran Flakes $(60 \mathrm{~g} / \mathrm{d})$ on human gut microbiota and to evaluate the diversity of the microbial community and any changes in major bacterial groups, using in situ molecular probe methodology. Also, the study measured changes in faecal short chain fatty acids. A further important objective was to confirm the effects of high-fiber cereals on fatigue, cognition and digestive functioning as assessed through the use of daily and 14-day questionnaires.

\section{Materials and Methods}

\subsection{All-Bran, Bran Flakes and Cornflakes}

The three breakfast cereals, All-Bran, Bran Flakes and Cornflakes were provided by Kellogg's UK (Manchester) in plain white boxes and all were given at $60 \mathrm{~g} / \mathrm{d}$, which are typical servings. On arrival, the boxes were stored at ambient temperature until required for the human volunteer study. The fiber in the bran products is wheat derived while it is corn for the corn flakes. Approximate levels in these servings would be All-Bran (20g/d), Bran Flakes $(10 \mathrm{~g} / \mathrm{d})$ and Cornflakes $(2 \mathrm{~g} / \mathrm{d})$. The nutrient composition of the cereals were (per 30g serving):

\section{1) All Bran: Calories: 80, Total Fat: 1g, Sodium: 80mg, Sugars: 6g, Dietary Fiber: 10g'}

Wheat bran, sugar, malt flavor, contains $2 \%$ or less of salt. Vitamins and Minerals: Calcium carbonate, vitamin C (sodium ascorbate and ascorbic acid), reduced iron, niacinamide, vitamin B6 (pyridoxine hydrochloride), vitamin B2 (riboflavin), folic acid, vitamin B1 (thiamin hydrochloride), vitamin A palmitate, vitamin B12, vitamin D.

\section{2) Bran flakes: Calories: 90, Total Fat: 0.5g, Sodium: 210mg, Sugars: $5 \mathrm{~g}$, Dietary Fiber: $5 \mathrm{~g}$}

Whole grain wheat, wheat bran, sugar, brown sugar syrup, contains $2 \%$ or less of salt, malt flavoring, BHT for freshness. Vitamins and Minerals: Vitamin C (sodium ascorbate, ascorbic acid), vitamin A palmitate, niacinamide, reduced iron, zinc oxide, calcium pantothenate, vitamin B6 (pyridoxine hydrochloride), vitamin D, vitamin B2 (riboflavin), vitamin B1 (thiamin hydrochloride), vitamin E (alpha tocopherol acetate), folic acid, vitamin B12.

3) Corn flakes: Calories: 100, Total Fat: 0g, Sodium: 200mg, Sugars: 3g, Dietary Fiber: 1g

Corn fiber, potassium, sodium, sugar, protein, vitamin A, vitamin C, vitamin B12, vitamin B6, iron, calcium, magnesium, vitamin D.

\subsection{Characteristics of the Volunteers}

A total of 20 volunteers were recruited from the staff and students of the University of Reading. The study number was based upon to detecting $0.2 \log _{10}$ change in bifidobacterial counts with power set at 0.9 , and a significance level of 0.05 , with bifidobacteria being the usual target genus for prebiotic effects. The main characteristics of the study population were:

- Gender: 12 females and 8 males

- Age: $25-45$ years

- Diet type: 17 non-vegetarian and 3 vegetarian

- Body mass index: 20-30 inclusive

- General health: good 
During the selection of the volunteers the following specific exclusion criteria were employed:

- Any requirement to take long-term medication (e.g. those active on the GI tract)

- Use of antibiotics within the previous 6 months

- History of alcohol or drug abuse

- Pregnancy or lactation or planning pregnancy

- Involvement in a study involving an experimental drug/medication within four weeks prior to entry to the study

- History of chronic constipation or diarrhoea, or other chronic GI complaint (e.g. irritable bowel syndrome, inflammatory conditions)

- Intake of other specific prebiotics or probiotics, drugs active on GI motility, or a laxative of any class within the four weeks prior to the start of the study

Informed consent forms were signed by each of the volunteers during a pre-trial assessment period after the volunteers had read the volunteer information sheets and had time to ask any questions about the study. The following specific requirements for diet and medication were made of the volunteers during the course of the study:

- No intake of additional breakfast cereals, prebiotics (e.g. fructo-oligosaccharides), probiotics (e.g. live yogurts), drugs active on GI motility, antibiotic treatment or any class of laxative

- All concomitant medication must be recorded in diaries and case record forms

- Usual diet or fluid intake should not be altered during the study period

\subsection{Ethical Review}

The project was approved by the Ethics and Research Committee of the University of Reading.

\subsection{Study Design}

The study used a cross-over design with three breakfast cereals (All-Bran, Bran Flakes and Cornflakes) and three experiment periods. The study was double blind and placebo controlled (i.e. Cornflakes). Washout periods of seven days occurred between the experimental periods in order to prevent any carry-over effect(s) from the previous condition.

The overall outline of the human volunteer study is given in Table 1. The study lasted a total of 10 weeks and involved both a baseline and an experimental phase. During the baseline period, lasting 14 days, and during the seven-day washout periods the volunteers were requested to refrain from consuming any form of breakfast cereal.

Table 1. Outline of human study.

\begin{tabular}{|c|c|c|}
\hline Day of study & Stage of study & Action \\
\hline-7 & Baseline & Provide base-line faecal sample (1) \\
\hline 0 & Start of study & $\begin{array}{l}\text { Distribution of test samples for the } 1^{\text {st }} 14 \text { day condition } \\
\text { Provide base-line faecal sample ( } 2)\end{array}$ \\
\hline $1-14$ & $1^{\text {st }}$ cereal period & $\begin{array}{l}\text { Take cereal sachets } \\
\text { Provide faecal samples on day } 7 \text { and } 14 \text { ( } 3 \text { and } 4)\end{array}$ \\
\hline $15-21$ & $1^{\text {st }}$ washout period & $\begin{array}{l}\text { No prebiotic to be taken on day } 15-21 \\
\text { Provide faecal sample on day } 21 \text { (5) } \\
\text { Distribution of test sachets on day } 21 \text { for the } 2^{\text {nd }} 14 \text { day period }\end{array}$ \\
\hline $22-35$ & $2^{\text {nd }}$ cereal period & $\begin{array}{l}\text { Take cereal sachets } \\
\text { Provide faecal sample on day } 28 \text { and } 35 \text { ( } 6 \text { and } 7)\end{array}$ \\
\hline $36-42$ & $2^{\text {nd }}$ washout period & $\begin{array}{l}\text { No prebiotic to be taken day } 36-42 \\
\text { Provide faecal sample on day } 42(8) \\
\text { Distribution of test sachets on day } 42 \text { for } 3^{\text {rd }} \text { period }\end{array}$ \\
\hline $43-56$ & 3rd cereal period & $\begin{array}{l}\text { Take cereal sachets } \\
\text { Provide faecal sample on day } 49 \text { and } 56 \text { (9 and 10) }\end{array}$ \\
\hline
\end{tabular}

Over the course of the study, each volunteer received all three breakfast cereals but not in the same order. The volunteers were randomly allocated to the conditions according to an incomplete Latin Square. The identification of the breakfast cereal conditions was blinded until completion of the study and to the volunteers immediately prior to the start of each condition when the boxes of breakfast cereals were distributed. 


\subsection{Faecal Sample Collection}

Throughout the study, faecal samples were collected at seven day intervals ( \pm one day, total of 10 faecal samples per volunteer). These were collected in sterile plastic pots, lined with a plastic bag, and stored at approximately $4^{\circ} \mathrm{C}$, and processed within two hours of collection.

\subsection{Faecal Analysis: Bacterial Enumeration}

Freshly voided faeces was diluted 1 in $10(\mathrm{w} / \mathrm{w})$ with anaerobic phosphate buffer $(0.1 \mathrm{M}, \mathrm{pH} 7.0)$ and homogenized in a stomacher at normal speed for $2 \mathrm{~min}$. Approximately $5-7 \mathrm{ml}$ of the faecal homogenate was transferred to a $50 \mathrm{ml}$ centrifuge tube containing $\sim 5 \mathrm{~mm}$ of glass beads and then vortex mixed for $\sim 30 \mathrm{~s}$. The tubes were centrifuged at $1500 \mathrm{xg}$ for $2 \mathrm{~min}$, to remove particulate matter, and then $375 \mu \mathrm{l}$ of the supernatant was transferred in duplicate to $2.5 \mathrm{ml}$ micro-centrifuge tubes containing $1125 \mu \mathrm{l}$ filtered $4 \%$ paraformaldehyde (previously chilled to $\sim 4^{\circ} \mathrm{C}$ ). The tubes contents were well mixed by inversion and the samples fixed during overnight storage at approximately $4^{\circ} \mathrm{C}$. After the lapsed time, $1.5 \mathrm{ml}$ of the fixed cells was transferred using a pipette to a sterile $2.5 \mathrm{ml}$ micro-centrifuge tube and then centrifuged at $13000 \mathrm{xg}$ for $5 \mathrm{~min}$. The supernatant was carefully removed using a pipette and then the pellet re-suspended in $1 \mathrm{ml}$ phosphate buffer. The tube was re-centrifuged at $13000 \mathrm{xg}$ for $5 \mathrm{~min}$ and then the pellet was washed for a second time in phosphate buffer. After carefully removing the supernatant, the pellet was finally re-suspended in $150 \mu 1$ filtered phosphate buffer and then $150 \mu \mathrm{l}$ of ethanol was added. The tube contents were thoroughly mixed before storing at $-20^{\circ} \mathrm{C}$.

The nucleic acid stain 4'6-diamidino-2-phenylindole (DAPI) was used for total bacterial counts. Enumeration of faecal bacterial populations was assessed through the use of the fluorescent in situ hybridisation (FISH) technique using molecular probes targeting specific diagnostic regions of $16 \mathrm{~S}$ rRNA. The predominant components of the gut microflora (bacteroides, bifidobacteria, clostridia, lactobacilli and eubacteria) were enumerated using genotypic probes tagged with fluorescent markers. Specifically, the probes used were Bif164 (Langendijk et al., 1995), Bac303 (Manz, Amann, Ludwig, Vancanneyt \& Schleiffer, 1996), His150 (Franks et al., 1998), Lab158 5 GGTATTAGCA(T/C)CTGTTTCCA (Harmsen Elfferich, Schut \& Welling, 1999) and Erec482 (Franks et al., 1998), specific for bifidobacteria, bacteroides, clostridia (Clostridium perfringens/histolyticum sub-group), lactobacilli/enterococci, and Eubacterium rectale/Clostridium coccoides sub-group respectively. Probes were commercially synthesized and 5' labelled with the fluorescent dye Cy3.

The cell suspension, following equilibration to ambient temperature for about $10 \mathrm{~min}$, was then added to the hybridisation buffer (ratio $16 \mu \mathrm{l}: 264 \mu \mathrm{l}$ for cell suspension and hybridisation buffer respectively). The hybridisation mixture was then added to the probes $(45 \mu 1: 5 \mu \mathrm{l}$ respectively) and hybridisation carried out, overnight, at an appropriate temperature for the probes (i.e. $50^{\circ} \mathrm{C}$ for total bacteria using DAPI, bifidobacteria, clostridia and eubacteria, $45^{\circ} \mathrm{C}$ for bacteroides and lactobacilli/enterococci). Subsequently, probes were filtered under vacuum, washed with hybridisation buffer and the filter mounted onto a microscope slide. Enumeration of bacteria was carried out using fluorescent microscopy.

\subsection{Fermentation Characteristics}

The faecal homogenate (dilution 1 in $10(\mathrm{w} / \mathrm{w})$ of faeces with anaerobic phosphate buffer) described above was also used for the determination of residual short chain fatty acid (SCFA) content by gas chromatography (GC). Centrifugation at $13000 \mathrm{xg}$ for $5 \mathrm{~min}$ was used to remove the particulate matter from $\sim 2 \mathrm{ml}$ faecal homogenate. The supernatant was then carefully transferred using a pipette to a clean $1.5 \mathrm{ml}$ micro-centrifuge tube and the sample stored frozen until required for subsequent SCFA analysis.

Following defrosting of the faecal supernatant samples at room temperature, the tubes were re-centrifuged at 13 $000 \mathrm{xg}$ for $5 \mathrm{~min}$ in order to remove any remaining particulate matter. Using a pipette, $0.8 \mathrm{ml}$ of the supernatant was then transferred to GC sample vial containing $0.2 \mathrm{ml}$ of 2-methyl-valeric acid (internal standard, $18.91 \mathrm{~g} / 1$ oxalic acid, $7.50 \mathrm{~g} / \mathrm{l}$ 2-methyl-valeric acid). The tube contents were mixed well and left overnight at approximately $4^{\circ} \mathrm{C}$. Standards were also prepared for each of the SCFAs (acetic, propionic, iso-butyric, n-butyric, iso-valeric and $\mathrm{n}$-valeric acids) in $0.03 \mathrm{M}$ oxalic acid. The supernatant samples and SCFA standards were then analysed by GC under the following conditions:

\begin{tabular}{lll}
\hline$\bullet$ & Column: & SGE 25 QC5/BP21 0.5, length $25 \mathrm{~m}$ \\
\hline$\bullet$ & Carrier gas: & Helium (Premier grade), Average flow rate $3 \mathrm{ml} / \mathrm{min}$ \\
\hline$\bullet$ & Injection: & Temperature set at $180^{\circ} \mathrm{C}$ \\
\hline$\bullet$ & Detection: & FID detector, detector temperature set at $180^{\circ} \mathrm{C}$ \\
\hline$\bullet$ & Temperature programme: & $\begin{array}{l}\text { Initial temperature } 85^{\circ} \mathrm{C}, \text { ramp rate } 6^{\circ} \mathrm{C} / \mathrm{min}, \\
\text { final oven temperature } 180^{\circ} \mathrm{C}\end{array}$ \\
\hline
\end{tabular}


The total run time was 15.8 min per sample for the elution of all acids and the internal standard. All results were initially recorded as mg SCFAs/ml. Results were used to calculate the mg SCFAs/g fresh faeces and the contribution/proportion of each SCFA to the total SCFA content of the faeces.

\subsection{Effects on Fatigue, Cognition and Digestive Functioning}

Volunteers completed a battery of questionnaires to assess fatigue/energy, subjective mood, physical and mental health, bowel function and fiber intake (Smith, 2005, 2010, 2011). During the three cereal conditions, volunteers completed both a daily questionnaire (for 14 days per period) and an overall 14-day questionnaire. On a daily basis, volunteers recorded their level of fatigue, mood and bowel function for that day. This was assessed using the daily questionnaire comprising 32 questions and was completed in the early evening of each day for each phase. The overall 14-day questionnaire was completed at the end of each condition.

\subsection{Statistical Analysis}

Analyses of variance were carried out on the bacteria enumeration, SCFA and questionnaire data using a General Linear Model. The data were subjected to extensive analysis in order to determine any effects of period or cereal on the parameters of interest. The approach to the analysis included the following:

- Analysis of the data from the baseline and washout periods alone (control data - no cereal effects)

- Analysis of the data from the three cereal periods alone

- Analysis of cereal data adjusted for the potential effect of the control data (i.e. cereal - baseline data and cereal/baseline data)

- Analysis of the cereal data using the baseline data as a covariate

\section{Results}

\subsection{Diversity of Faecal Bacterial Community}

Results showed that faecal bacterial numbers (as $\log _{10}$ cells/g fresh faeces) varied significantly between the volunteers employed at the start of the study. This observation confirmed that there was considerable variation in the bacterial populations of the human GI tract. It was the effect of volunteer, rather than a result of the cereal effects, that accounted for most of the differences observed in bacterial numbers during the course of the study. There was a significant effect of experimental period on four of the bacterial groups studied. This observation confirms that the GI tract is a dynamic system and that changes in bacterial numbers occur with time. This finding emphasises the importance of completing the present study as a Latin Square design in order to account for such variations throughout the period of the study.

With one exception, there was no significant effect of breakfast cereal on individual faecal bacterial populations studied (see Table 2).

Table 2. Effect of different breakfast cereals on faecal bacterial numbers (as $\log _{10}$ cells/g fresh faeces)

\begin{tabular}{|c|c|c|c|c|c|}
\hline Bacterial & Cereal & & & & \\
\hline Group & All-Bran & Bran Flakes & Cornflakes & Sig. & SED \\
\hline Total bacteria (DAPI) & 10.69 & 10.72 & 10.70 & $\mathrm{~ns}$ & 0.019 \\
\hline bifidobacteria & 9.26 & 9.31 & 9.28 & ns & 0.055 \\
\hline bacteroides & 9.98 & 9.99 & 9.99 & $\mathrm{~ns}$ & 0.040 \\
\hline lactobacilli/enterococci & 8.28 & 8.28 & 8.41 & $\mathrm{~ns}$ & 0.080 \\
\hline clostridia $^{1}$ & 8.41 & 8.19 & 8.49 & $*$ & 0.120 \\
\hline eubacteria $^{2}$ & 10.10 & 10.14 & 10.07 & $\mathrm{~ns}$ & 0.034 \\
\hline
\end{tabular}

Note. Sig., Statistical significance, SED, Standard error of difference, ${ }^{1}$, Clostridium perfringens/histoliticum sub-group, ${ }^{2}$, Eubacterium rectale/Clostridium coccoides sub-group, ${ }^{*}, \mathrm{p}<0.05$, ns, not significant (p $>0.05$ ).

A significant difference $(\mathrm{p}<0.05)$ in the numbers of the Clostridium perfringens/histolyticum sub-group was observed between Bran Flakes and Cornflakes (becoming reduced in the former condition). The absence of any significant effect of the cereal conditions on the remaining bacterial populations studied, for example bifidobacteria, may reflect the level of the bacteria in the study population (volunteers) used. For example, Tuohy, Kolida, Lustenberger and Gibson (2001) showed that there was no major effect of consuming experimental biscuits (providing $6.6 \mathrm{~g}$ fructo-oligosaccharides/d and $3.4 \mathrm{~g}$ partially hydrolysed guar gum/d) on the number of bifidobacteria in volunteers with initial levels of $9.45 \log _{10}$ cells/g faeces, i.e. high starting populations elicit little or no further effect. The other explanation is that the cereals did not act as prebiotics. 


\subsection{Short Chain Fatty Acids}

Short chain fatty acids, principally acetate, propionate and butyrate are the main metabolic end products of bacterial fermentation. However, measurements of SCFAs in faeces are only indicative of the amount of residual organic acids since the vast majority are rapidly absorbed or metabolised in the gut. Results showed that the mean total SCFA content of the faeces was low $(6.60 \mathrm{mg} / \mathrm{g}$ fresh faeces) and that the mean proportion of acetate, propionate and n-butyrate was $0.48,0.21$ and 0.22 respectively. No fluctuations in response to the individual cereals was observed.

Faecal SCFA content (mg/g fresh faeces) and the proportion of SCFAs varied significantly between the volunteers. The results given in Table 3 show that there was no significant effect of breakfast cereal on either the total or individual SCFA content expressed as mg SCFA/g fresh faeces. When the individual SCFAs were expressed as a proportion of the total, the results showed a significant effect of cereal on i-valerate $(\mathrm{p}<0.01)$ and $\mathrm{n}$-valerate $(\mathrm{p}<0.05)$. Although statistically significant, both of these acids accounted for less than 0.07 of the total SCFA measured in the faeces (equivalent to approximately $0.45 \mathrm{mg} / \mathrm{g}$ fresh faeces). The results are not viewed to be biologically relevant, therefore.

Table 3. Effect of breakfast cereals on SCFA in faeces

\begin{tabular}{|c|c|c|c|c|c|}
\hline \multicolumn{6}{|c|}{ Cereal } \\
\hline Parameter & All-Bran & Bran Flakes & Cornflakes & Sig. & SED \\
\hline \multicolumn{6}{|c|}{ SCFA content ( $\mathrm{mg} / \mathrm{g}$ fresh faeces) } \\
\hline Acetate & 3.32 & 2.98 & 3.11 & $\mathrm{~ns}$ & 0.232 \\
\hline Propionate & 1.39 & 1.26 & 1.41 & ns & 0.092 \\
\hline i-Butyrate & 0.144 & 0.155 & 0.162 & ns & 0.0174 \\
\hline n-Butyrate & 1.72 & 1.48 & 1.40 & $\mathrm{~ns}$ & 0.146 \\
\hline i-Valerate & 0.203 & 0.251 & 0.261 & $\mathrm{~ns}$ & 0.0281 \\
\hline $\mathrm{n}$-Valerate & 0.170 & 0.185 & 0.198 & $\mathrm{~ns}$ & 0.0182 \\
\hline Total & 6.94 & 6.30 & 6.55 & ns & 0.394 \\
\hline \multicolumn{6}{|c|}{ SCFA proportions } \\
\hline Acetate & 0.489 & 0.476 & 0.480 & $\mathrm{~ns}$ & 0.0140 \\
\hline Propionate & 0.206 & 0.201 & 0.214 & $\mathrm{~ns}$ & 0.0088 \\
\hline i-Butyrate & 0.021 & 0.025 & 0.027 & $\mathrm{~ns}$ & 0.0024 \\
\hline n-Butyrate & 0.227 & 0.226 & 0.205 & $\mathrm{~ns}$ & 0.0112 \\
\hline i-Valerate & 0.031 & 0.043 & 0.044 & ** & 0.0046 \\
\hline n-Valerate & 0.026 & 0.029 & 0.031 & * & 0.0023 \\
\hline
\end{tabular}

Note. Sig., Statistical significance, SED, Standard error of difference, SCFA, Short chain fatty acid, *, P $<0.05$, ns, not significant $(\mathrm{P}>0.05)$.

\subsection{Effects of High-fiber Cereal on Fatigue, Cognition and Digestive Functioning}

Following Smith et al. (2001), high fiber cereals (All Bran + Bran flakes) were compared with the cornflakes. Results from the 14 day questionnaires showed that high fiber was associated with less fatigue (High fiber: mean=23.7 s.e. $=2.8$, Cornflakes $=25.8$ s.e. $=3.2)$. This effect was not significant $(p=0.16)$ which reflects the smaller study population size used in the present study as compared to that used by Smith et al. (2001). However, high fiber cereal intake was associated with a significant reduction in cognitive difficulties (High fiber: mean $=19.1$, s.e. $=1.9$, Cornflakes: mean $=22.5$ s.e. $=2.4, \mathrm{p}<0.05$ ).

Analyses of the digestive symptoms showed that the high-fiber cereal conditions were associated with looser stools, stomach pains, and wind and less incomplete evacuation of the bowels (all p's $<0.05$ ). While these effects were significant, the magnitude of the effects was small and we are essentially comparing no symptoms with very slight symptoms. Overall, the pattern of results confirms those obtained previously by Smith et al. (2001). Analysis of the daily diaries showed that the high-fiber cereal conditions were associated with looser stools, more motions and feeling more energised (all p's $<0.05$ ).

\section{Discussion}

The major aims of the present human study were to confirm that consumption of wheat bran cereals increase energy (reduce fatigue) and reduce cognitive difficulties. A secondary aim was to determine whether wheat bran cereals have a prebiotic effect that could explain behavioural effects associated with consumption of these cereals. High-fiber conditions (All-Bran and Bran Flakes) were associated with less fatigue and feeling more 
energised. These findings are in agreement with those reported previously by Smith et al. (2001). Smith (2005) suggested that fiber is fermented to short chain fatty acids by gut microbiota. Their metabolites may then have systemic effects, e.g. acetate in muscle tissues generates ATP and therefore energy, butyrate is metabolized by colonocytes. Gut fermentation and subsequent use of SCFA may contribute up to $10 \%$ of a person's energy requirements (Cummings \& Macfarlane, 1997). This explanation plausibly accounts for the rapid effect of fiber and the subsequent effect. The second possible mechanism could be due to the components of microbiota that are stimulated, e.g. many clostridia are known to form toxins, while prebiotic fiber enhances benign microflora (bifidobacteria, lactobacilli) which are not pathogenic (Gibson et al., 2017). In the present study changes in fatigue and cognitive function were generally not associated with major changes in either the diversity of the faecal microflora or the residual SCFA content of the faeces as a result of the breakfast cereals. One exception was that the Clostridium perfringens/histolyticum sub-group was significantly lower in volunteers consuming Bran Flakes than when consuming Cornflakes, however there was no significant difference between the All-Bran and Cornflakes. Clostridia are generally considered to be negative for health and this observation with Bran is a positive outcome from the study. However, the cereals did not elicit changes in beneficial genera such as bifidobacteria. It may therefore be necessary to fortify with prebiotics to generate a more positive influence on the gut microbiota.

Any changes in bacterial populations or faecal SCFAs were largely accounted for by highly significant differences between volunteers, and also period of the experiment (i.e. changes over time). In order to observe differences in the measured parameters, the results suggest that a larger population size is required and/or that the population be stratified according to particular characteristics (e.g. sex (all female or male), age, diet type). Such criteria could be selected from the results of preference testing completed on the products of interest or individual variation in the gut flora. Similarly, further work is required to determine the effect of altering the processing method(s) of the high-fiber breakfast cereals on their intrinsic prebiotic potential. This would be an alternative to straightforward prebiotic supplementation. For example, further work could be carried out to determine the effect of using enzymes (e.g. xylanases) to increase the prebiotic potential of the high-fiber breakfast cereals. It is important to investigate whether increased prebiotic effects of cereals (by supplementation or tailoring of processing approaches) has benefits beyond those seen with the cereal itself. The data shown here shows promise in this regard, from the viewpoint of both cognitive and GI issues.

\section{Acknowledgements}

This research was funded by the Kellogg's Company Manchester and we would like to thank Reg Fletcher for his contribution to the study. The sponsors had no role in the collection, analyses or interpretation of data or writing the manuscript.

\section{Conflict of interest}

The authors declare no conflict of interest.

\section{References}

Belsey, J., Greenfield, S., Candy, D., \& Geraint, M. (2010). Systematic review: Impact of constipation on quality of life in adults and children. Alimentary Pharmacology \& Therapeutics. 31(9), 938-949. https://doi.org/10.1111/j.1365-2036.2010.04273.x

Cummings, J. H., \& Macfarlane, G. T. (1997). Colonic microflora: nutrition and health. Nutrition, 13, 476-478. https://doi.org/10.1016/S0899-9007(97)00114-7

Franks, A. H., Harmsen, H. J. M., Raangs, G. C., Jansen, G. J., Schut, F., \& Welling, G. W. (1998). Variations of bacterial populations in human faeces measured by fluorescent in situ hybridization with group-specific $16 \mathrm{~S}$ rRNA-targeted oligonucleotide probes. Applied and Environmental Microbiology, 64, 3336-3345. https://doi.org/10.12938/bifidus1996.19.79

Garrow, J. S., \& James, W. P. T. (1993). Human Nutrition and Dietetics( 9th ed.). Edinburgh, United Kingdom: Churchill Livingstone.

Gibson, G. R. (1998). Dietary modulation of the human gut microflora using prebiotics. British Journal of Nutrition, 80(4), S209-S212.

Gibson, G. R., Hutkins, R., Sanders, M. E., Prescott, S. L., Reimer, R. A., Salminen, S. J., .. Reid, G. (2017). Expert consensus document: The International Scientific Association for Probiotics and Prebiotics (ISAPP) consensus statement on the definition and scope of prebiotics. Nature Reviews in Gastroenterology \& Hepatology, 14(8), 491-502, https://doi.org/10.1038/nrgastro.2017.75. 
Gibson, G. R., \& Roberfroid, M. B. (1995). Dietary modulation of the human colonic microbiota: introducing the concept of prebiotics. Journal of Nutrition, 125(6), 1401-1412.

Gray, D. S. (1995). The clinical uses of dietary fiber. American Family Physician, 51(2), 419-426.

Harmsen, H. J. M, Elfferich, P., Schut, F. \& Welling G. W. (1999). A 16S rRNA-targeted probe for detection of lactobacilli and enterococci in faecal samples by fluorescent in situ hybridization. Microbial Ecology in Health and Disease, 11, 3-12.

Hill, C., Guarner, F., Reid, G., Gibson, G. R., Merenstein, D. J., Pot, B., ... Sanders, M.E. (2014). Expert consensus document: The International Scientific Association for Probiotics and Prebiotics consensus statement on the scope and appropriate use of the term probiotic. Nature Reviews in Gastroenterology \& Hepatology, 11(8), 506-514. https://doi.org/10.1038/nrgastro.2014.66.

Hull, C., Greco, R. S., \& Brooks, D. L. (1980). Alleviation of constipation in the elderly by dietary fiber supplementation. Journal of the American Geriatrics Society, 28(9), 410-414. https://doi.org/10.1111/j.1532-5415.1980tb01108.x

Langendijk, P.S., Schut, F., Jansen, G. J., Raangs, G. W., Kamphuis, G. R., Wilkinson, M. H. F., \& Welling, G. W. (1995). Quantitative fluorescent in situ hybridization of Bifidobacterium spp. with genus-specific 16S rRNA-targeted probes and its application in faecal samples. Applied and Environmental Microbiology., 61(8), 3069-3075.

Manz, W., Amann, R., Ludwig, W., Vancanneyt, M. \& Schleiffer, K. H. (1996). Application of a suite of 16S rRNA-specific oligonucleotide probes designed to investigate bacteria of the phylum cytophaga-flavobacter-bacteroides in the natural environment. Microbiology, 142, 1097-1106, https://doi.org/10.1099/13500872-142-5-1097.

Merkel, I. S., Locher, J., Burgio, K., Towers, A., \& Wald, A. (1993). Physiologic and psychologic characteristics of an elderly population with chronic constipation. American Journal of Gastroenterology, 88(11), 1854-1859.

Smith, A. P. (2005). The concept of well-being: relevance to nutritional research. British Journal of Nutrition, 93(S1), S1-S5, https://doi.org/10.1079/BJN20041351

Smith, A. P. (2010). Breakfast cereal, fiber, digestive problems and well-being. Current Topics in Nutraceutical Research, 8, 117-126, https://doi.org/10.1002/smi.1390

Smith, A. P. (2011). Breakfast cereal, digestive problems and well-being. Stress Health, 27(5), 388-394, https://doi.org/10.1002/smi.1390.

Smith, A. P., Bazzoni, C., Beale, J., Elliot-Smith, J., Tilley, M. (2001). High fiber breakfast cereals reduce fatigue. Appetite, 37(3), 249-250, https://doi.org/10.1006/appe.2001.0428

Taylor, R. (1990). High fiber diet works. British Medical Journal, 300(6731), 1063-1064.

Tuohy, K. M., Kolida, S., Lustenberger, A. M., \& Gibson, G. R. (2001). The prebiotic effect of biscuits containing partially hydrolysed guar gum and fructo-oligosaccharides - a human volunteer study. British Journal of Nutrition, 86(3), 341-348. https://doi.org/10.1079/BJN2001394.

\section{Copyrights}

Copyright for this article is retained by the author(s), with first publication rights granted to the journal.

This is an open-access article distributed under the terms and conditions of the Creative Commons Attribution license (http://creativecommons.org/licenses/by/4.0/). 